\title{
APPLICATIONS OF ABSTRACT PARABOLIC QUASI-VARIATIONAL INEQUALITIES TO OBSTACLE PROBLEMS
}

\author{
RISEI KANO \\ Department of Mathematics, Graduate School of Science and Technology \\ Chiba University \\ 1-33 Yayoi-cho, Inage-ku, Chiba, 263-8522 Japan \\ E-mail:kano@hiro.kindai.ac.jp
}

\begin{abstract}
In this paper we study a class of abstract quasi-variational inequalities with nonlocal constraints depending on the unknown and establish an existence result. Further we give its applications to parabolic systems of partial differential inequalities with nonlocal obstacles depending on the unknowns.
\end{abstract}

1. Introduction. For a real Hilbert space $H$, we are given a family $\left\{\varphi^{t}(v ; \cdot)\right\}$ of proper, lower semicontinuous, convex functions $\varphi^{t}(v ; \cdot)$ on $H$, with parameters $t \in[0, T]$ for a fixed finite time $T$ and $v$ in an appropriate function space, and consider a nonlinear evolution equation of the form:

$$
u^{\prime}(t)+\partial \varphi^{t}(u ; u(t)) \ni f(t), \quad 0<t<T, \text { in } H,
$$

subject to the initial condition

$$
u(t)=u_{0}(t), \quad-\delta_{0} \leq t \leq 0, \text { in } H,
$$

where $0<\delta_{0}<\infty, \partial \varphi^{t}(u ; \cdot)$ is the subdifferential of convex function $\varphi^{t}(u ; \cdot)$ on $H$, $u^{\prime}=\frac{d u}{d t}$ and $u_{0}:\left[-\delta_{0}, 0\right] \rightarrow H$ and $f:(0, T) \rightarrow H$ are prescribed as initial and forcing functions, respectively. In this paper, we shall prove that the above Cauchy problem has at least one solution $u$ which is local or global in time.

There are a lot of results for elliptic quasi-variational inequalities (cf. $[1,5])$, but the corresponding parabolic problems are extremely difficult to handle. In this paper we propose a mathematical approach to the parabolic case in an abstract framework.

2000 Mathematics Subject Classification: Primary 35K45; Secondary 35K50.

Key words and phrases: parabolic, quasi-variational inequality, subdifferential, PDE. The paper is in final form and no version of it will be published elsewhere. 
As an example of the above abstract problem, we have the following system:

$$
\begin{aligned}
& u \in W^{1,2}\left(-\delta_{0}, T ; L^{2}(0,1)\right) \cap L^{\infty}\left(-\delta_{0}, T ; H^{1}(0,1)\right), \\
& \left|u_{x}\right| \leq k_{c}(\Lambda u) \text { a.e. on }(0,1) \times(0, T), \quad u(0, t)=0 \text { for } 0<t<T, \\
& \int_{0}^{T} \int_{0}^{1}\left(u_{t}-f\right)(u-w) d x d t+\int_{0}^{T} \int_{0}^{1} u_{x}\left(u_{x}-w_{x}\right) d x d t \leq 0, \\
& \forall w \in L^{2}\left(0, T ; H^{1}(0,1)\right) \text { with }\left|w_{x}\right| \leq k_{c}(\Lambda u) \text { a.e. on }(0,1) \times(0, T) \\
& \text { and } w(0, t)=0 \text { for } 0<t<T, \\
& u=u_{0} \text { on }(0,1) \times\left[-\delta_{0}, 0\right],
\end{aligned}
$$

where $k_{c}(\cdot)$ is a Lipschitz continuous function on $\mathbf{R}, f$ is a given function on $(0,1) \times(0, T)$ and $\Lambda$ is a nonlocal mapping defined by

$$
[\Lambda v](x, t):=\int_{-\delta_{0}}^{t} \int_{0}^{1} \rho(x, y, s, t) v(y, s) d y d s, \forall(x, t) \in(0,1) \times[0, T],
$$

where $\rho$ be a bounded smooth function on $[0,1] \times[0,1] \times\left[-\delta_{0}, T\right] \times[0, T]$. In this system (1.1) the constraint $k_{c}(\Lambda u)$ depends upon the unknown function $u$, so that this system is called a quasi-variational inequality.

2. Notation and known results. Let $X$ be a real reflexive Banach space, $X^{*}$ be its dual and $H$ be a real Hilbert space such that $X$ is dense in $H$ and the natural injection from $X$ into $H$ is compact:

$$
X \subset H \subset X^{*}
$$

We denote by $|\cdot|_{H},|\cdot|_{X}$ and $|\cdot|_{X^{*}}$ the norms in $H, X$ and $X^{*}$, respectively, and denote by $(\cdot, \cdot)_{H}$ the inner product in $H$.

Given a proper, lower semi-continuous (l.s.c.) and convex function $\psi$ on $H$, the effective domain of $\psi$ is the set

$$
D(\psi)=\{z \in H: \psi(z)<\infty\},
$$

and the subdifferential $\partial \psi$ of $\psi$ is a (multivalued) operator in $H$ defined by

$$
z^{*} \in \partial \psi(z) \Leftrightarrow z \in D(\psi),\left(z^{*}, y-z\right)_{H} \leq \psi(y)-\psi(z), \forall y \in H .
$$

Let us recall a notion of convergence of convex functions. Given a sequence $\left\{\psi_{n}\right\}$ of proper l.s.c. and convex functions on $H$, it is said that $\psi_{n}$ converges to a proper, l.s.c. and convex function $\psi$ on $H$ in the sense of Mosco [7], if the following two conditions (M1) and (M2) are fulfilled:

(M1) If $z_{n} \rightarrow z$ weakly in $H$, then $\liminf _{n \rightarrow \infty} \psi_{n}(z) \geq \psi(z)$.

(M2) For each $z \in D(\psi)$ there is a sequence $\left\{z_{n}\right\}$ in $H$ such that $z_{n} \rightarrow z$ in $H$ and $\psi_{n}\left(z_{n}\right) \rightarrow \psi(z)$ as $n \rightarrow \infty$.

Given non-negative functions $a \in L^{2}(0, T)$ and $b \in L^{1}(0, T)$, we define the class $G(a, b)$ of all families $\left\{\psi^{t}(\cdot)\right\}_{0 \leq t \leq T}$ consisting of proper, l.s.c. and convex functions on $H$ as follows: $\left\{\psi^{t}(\cdot)\right\}_{0 \leq t \leq T} \in G(a, b)$ if and only if the following condition (*) is fulfilled: 
(*) For any $s, t \in[0, T]$, and any $z \in D\left(\psi^{s}\right)$, there exists $\tilde{z} \in D\left(\psi^{t}\right)$ such that

$$
|\tilde{z}-z|_{H} \leq \int_{s}^{t} a(\tau) d \tau\left(1+\psi^{s}(z)^{\frac{1}{2}}\right)
$$

and

$$
\psi^{t}(\tilde{z})-\psi^{s}(z) \leq \int_{s}^{t} b(\tau) d \tau\left(1+\psi^{s}(z)\right)
$$

Let us consider an evolution problem, labelled (CP) or more precisely (CP; $\left.\left\{\psi^{t}\right\}, u_{0}, f\right)$, generated by the subdifferential $\partial \psi^{t}$ :

$$
(\mathrm{CP})\left\{\begin{array}{l}
u^{\prime}(t)+\partial \psi^{t}(u(t)) \ni f(t) \text { a.e. } t \in[0, T], \\
u(0)=u_{0}
\end{array}\right.
$$

where $f$ and $u_{0}$ are respectively prescribed in $L^{2}(0, T ; H)$ and $H$. We say that $u$ is a solution of $(\mathrm{CP})$ on $[0, T]$, if $u \in C([0, T] ; H) \cap W_{l o c}^{1,2}((0, T] ; H), \psi^{(\cdot)}(u) \in L^{1}(0, T)$, $u(0)=u_{0}$ and $f(t)-u^{\prime}(t) \in \partial \psi^{t}(u(t))$ for a.e. $t \in(0, T)$.

On account of $[3,4,10]$, if $\left\{\psi^{t}\right\}_{0 \leq t \leq T} \in G(a, b), u_{0} \in D\left(\psi^{0}\right)$ and $f \in L^{2}(0, T ; H)$, then (CP) has a unique solution $u$ such that $u \in W^{1,2}(0, T ; H)$ and $\sup _{0 \leq t \leq T} \psi^{t}(u(t))<\infty$. Furthermore, we have

$$
\begin{gathered}
\frac{1}{2}|u(t)|_{H}^{2}+\int_{0}^{t} \psi^{\tau}(u(\tau)) d \tau-(u(t), h(t))_{H}+\int_{0}^{t}\left(u(\tau), h^{\prime}(\tau)\right)_{H} d \tau-\int_{0}^{t}(f(\tau), u(\tau))_{H} d \tau \\
\leq \frac{1}{2}\left|u_{0}\right|_{H}^{2}+\int_{0}^{t} \psi^{\tau}(h(\tau)) d \tau-\left(u_{0}, h(0)\right)_{H}-\int_{t}^{0}(f(\tau), h(\tau))_{H} d \tau, \forall t \in[0, T],
\end{gathered}
$$

for any function $h \in W^{1,2}(0, T ; H)$ with $\psi^{(\cdot)}(h(\cdot)) \in L^{1}(0, T)$, and

$$
\psi^{t}(u(t))+\frac{1}{2} \int_{0}^{t}\left|u^{\prime}(\tau)\right|_{H} d \tau \leq \psi^{0}\left(u_{0}\right)+\int_{0}^{t} k(\tau) \cdot\left(\psi^{\tau}(u(\tau))+1\right) d \tau, \forall t \in[0, T],
$$

where $k(\cdot)=6\left\{a(\cdot)^{2}+b(\cdot)+|f(\cdot)|_{H}^{2}\right\}$.

Next, we recall a convergence theorem (cf. [4]) for the above problem (CP). Let $\left\{\psi_{n}^{t}\right\}$ be a sequence in $G(a, b)$, and $\left\{u_{0 n}\right\}$ and $\left\{f_{n}\right\}$ be sequences in $H$ and $L^{2}(0, T ; H)$, respectively, such that

(a) $\psi_{n}^{t}$ converges to $\psi^{t}$ on $H$ in the sense of Mosco as $n \rightarrow \infty$ for every $t \in[0, T]$, where $\left\{\psi^{t}\right\}$ is a family in $G(a, b)$.

(b) $u_{0 n} \in D\left(\psi_{n}^{0}\right), \sup _{n \in \mathbb{N}} \psi_{n}^{0}\left(u_{n}\right)<\infty, u_{0} \in D\left(\psi^{0}\right), u_{0 n} \rightarrow u_{0}$ in $H$ and $f_{n} \rightarrow f$ in $L^{2}(0, T ; H)$ as $n \rightarrow \infty$.

Then the solution $u_{n}$ of $\mathrm{CP}\left(\left\{\psi_{n}^{t}\right\}, u_{0 n}, f_{n}\right)$ converges to the solution $u$ of $\mathrm{CP}\left(\left\{\psi^{t}\right\}, u_{0}, f\right)$ in the sense that

$$
u_{n} \rightarrow u \text { in } C([0, T] ; H), u_{n}^{\prime} \rightarrow u^{\prime} \text { weakly in } L^{2}(0, T ; H),
$$

$$
\sup _{n \in \mathbb{N}, t \in[0, T]} \psi_{n}^{t}\left(u_{n}(t)\right)<\infty, \quad \psi_{n}^{t}\left(u_{n}(t)\right) \rightarrow \psi^{t}(u(t)) \text { for a.e. } t \in[0, T],
$$

and

$$
\int_{0}^{T} \psi_{n}^{t}\left(u_{n}(t)\right) d t \rightarrow \int_{0}^{T} \psi^{t}(u(t)) d t
$$


3. Existence results. Let $\delta_{0}$ and $T$ be fixed positive numbers as well as $2 \leq p<+\infty$, and put

$$
\begin{aligned}
& V\left(-\delta_{0}, t\right):=L^{\infty}\left(-\delta_{0}, t ; H\right) \cap L^{p}\left(-\delta_{0}, t ; X\right), 0 \leq t \leq T, \\
& |v|_{V\left(-\delta_{0}, t\right)}:=|v|_{L^{\infty}\left(-\delta_{0}, t ; H\right)}+|v|_{L^{p}\left(-\delta_{0}, t ; X\right)} .
\end{aligned}
$$

Now, to each $t \in[0, T]$ and each $v \in V\left(-\delta_{0}, t\right)$, a family $\left\{\varphi^{s}(v ; \cdot) ; 0 \leq s \leq t\right\}$ of functions $\varphi^{s}(v ; \cdot)$ from $H$ into $[0, \infty]$ is assigned such that

(\$1) $\varphi^{s}(v ; z)$ is proper, l.s.c., non-negative and convex in $z \in H$ and $\varphi^{s}(v ; z)$ is determined by the values of $v$ on $\left(-\delta_{0}, s\right)$, namely

$\varphi^{s}\left(v_{1} ; \cdot\right)=\varphi^{s}\left(v_{2} ; \cdot\right)$ on $H$, wherever $v_{1}, v_{2} \in V\left(-\delta_{0}, t\right)$ and $v_{1}=v_{2}$ a.e. on $\left(-\delta_{0}, s\right)$.

(Ф2) (Coerciveness) There is a positive constant $C_{0}$ such that

$$
\varphi^{s}(v ; z) \geq C_{0}|z|_{X}^{p}, \forall 0 \leq s \leq t \leq T, \forall v \in V\left(-\delta_{0}, t\right), \forall z \in H .
$$

(Ф3) (Continuity) If $0 \leq s_{n} \leq t \leq T, v_{n} \in V\left(-\delta_{0}, t\right), s_{n} \rightarrow s$ and $v_{n} \rightarrow v$ weakly in $L^{p}\left(-\delta_{0}, t ; X\right)$ and weakly* in $L^{\infty}\left(-\delta_{0}, t ; H\right)$, then $\varphi^{s_{n}}\left(v_{n} ; \cdot\right) \rightarrow \varphi^{s}(v ; \cdot)$ on $H$ in the sense of Mosco.

Now we consider the following abstract evolution equation

$$
\mathrm{CP}\left(u_{0}, f\right)\left\{\begin{array}{l}
u^{\prime}(t)+\partial \varphi^{t}(u ; u(t)) \ni f(t) \text { in } H, t \in(0, T), \\
u(t)=u_{0}(t) \text { in } H, \forall t \in\left[-\delta_{0}, 0\right],
\end{array}\right.
$$

where $f$ is a function given in $L^{2}(0, T ; H), u_{0} \in C\left(\left[-\delta_{0}, 0\right] ; H\right)$, and $\partial \varphi^{t}(u ; \cdot)$ is the subdifferential of $\varphi^{t}(u ; \cdot)$. We say that a function $u:\left[-\delta_{0}, T\right] \rightarrow H$ is a solution of $\mathrm{CP}\left(u_{0}, f\right)$ on $[0, T]$, if $u \in C\left(\left[-\delta_{0}, T\right] ; H\right) \cap W_{l o c}^{1,2}((0, T] ; H), \varphi^{(\cdot)}(u ; u(\cdot)) \in L^{1}(0, T), f(t)-$ $u^{\prime}(t) \in \partial \varphi^{t}(u ; u(t))$ for a.e. $t \in[0, T]$ and $u(t)=u_{0}(t)$ in $H$, for all $t \in\left[-\delta_{0}, 0\right]$.

In order to specify the dependence of $\varphi^{s}(v ; \cdot)$ upon $v$, we use the class $G(a, b)$ of proper, l.s.c., convex functions. Now we give our main results of this paper.

THEOREM 1. Assume that for each number $M>0$ there are non-negative functions $a_{M} \in L^{2}(0, T)$ and $b_{M} \in L^{1}(0, T)$ such that

(H1) $\left\{\varphi^{t}(v ; \cdot)\right\}_{0 \leq t \leq T} \in G\left(a_{M}, b_{M}\right)$, whenever $|v|_{V\left(-\delta_{0}, T\right)} \leq M$.

Let $f \in L^{2}(0, T ; H)$ and $u_{0} \in W^{1,2}\left(-\delta_{0}, 0 ; H\right) \cap L^{p}\left(-\delta_{0}, 0 ; X\right)$ be such that $\varphi^{0}\left(u_{0} ; u_{0}(0)\right)<$ $+\infty$. Then there exist $0<T^{\prime} \leq T$ and a solution $u$ of $\mathrm{CP}\left(u_{0}, f\right)$ on $\left[0, T^{\prime}\right]$ such that

$$
u \in W^{1,2}\left(-\delta_{0}, T^{\prime} ; H\right), \sup _{t \in\left[0, T^{\prime}\right]} \varphi^{t}(u ; u(t))<\infty .
$$

THEOREM 2. Assume that for each number $M>0$ there are non-negative functions $a_{M} \in L^{2}(0, T)$ and $b_{M} \in L^{1}(0, T)$ such that (H1) holds. Further assume that there is a constant $A_{0}>0$ such that

(H2) $\forall v \in V\left(-\delta_{0}, T\right), \exists h_{v} \in W^{1,2}(0, T ; H) \cap L^{p}(0, T ; X)$ such that

$$
\left|h_{v}\right|_{W^{1,2}(0, T ; H)} \leq A_{0}, \quad \int_{0}^{T} \varphi^{t}\left(v ; h_{v}(t)\right) d t \leq A_{0},
$$


Let $f \in L^{2}(0, T ; H)$ and $u_{0} \in W^{1,2}\left(-\delta_{0}, 0 ; H\right) \cap L^{p}\left(-\delta_{0}, 0 ; X\right)$ be such that $\varphi^{0}\left(u_{0} ; u_{0}(0)\right)<$ $+\infty$. Then there exists a solution $u$ of $\mathrm{CP}\left(u_{0}, f\right)$ on $[0, T]$ such that

$$
u \in W^{1,2}\left(-\delta_{0}, T ; H\right), \sup _{t \in[0, T]} \varphi^{t}(u ; u(t))<\infty .
$$

We shall prove Theorems 1 and 2 in the following sections 4 and 5 . Our third theorem gives an existence result for $\operatorname{CP}\left(u_{0}, f\right)$, when the initial datum $u_{0}$ is not good. The details of proof are presented in the paper [2].

THEOREM 3. Assume that for each number $M>0$ there are non-negative functions $a_{M} \in$ $L^{2}(0, T)$ and $b_{M} \in L^{1}(0, T)$ such that (H1) holds, and assume that there is a constant $A_{0}>0$ such that (H2) holds. Let $f \in L^{2}(0, T ; H)$ and $u_{0} \in C\left(\left[-\delta_{0}, 0\right] ; H\right) \cap L^{p}\left(-\delta_{0}, 0 ; X\right)$. Suppose that there exists $\left\{u_{0_{n}}\right\} \subset V\left(-\delta_{0}, 0\right)$ with $\varphi^{0}\left(u_{0_{n}} ; u_{0_{n}}(0)\right)<+\infty$, such that

$$
u_{0_{n}} \rightarrow u_{0} \text { in } C\left(\left[-\delta_{0}, 0\right] ; H\right) \text {, weakly in } L^{p}\left(-\delta_{0}, 0 ; X\right) \text {. }
$$

Then there exists a solution $u$ of $\mathrm{CP}\left(u_{0}, f\right)$ on $[0, T]$ such that

$$
u \in C([0, T] ; H), t^{\frac{1}{2}} u^{\prime} \in L^{2}(0, T ; H), t \varphi^{t}(u ; u(\cdot)) \in L^{\infty}(0, T) .
$$

As far as the existence of a solution of the quasi-variational evolution problem is concerned, we can work in a much larger class of $\left\{\varphi^{t}(v ; \cdot)\right\}$ than that of this paper; see [2] and also $[6,9]$. Actually our class of $\left\{\varphi^{t}(v ; \cdot)\right\}$ treated in this paper is a special case of that in [2]. However, from the view-point of applications we can say that our class is important because the existence proof is much simpler than that of [2] and many interesting examples arising in physics and mechanics are included in this class. This is the reason why the proof of existence result is given in this paper, although it is a special case of the general theory.

4. Proof of Theorem 1. Let $M_{0}=\varphi^{0}\left(u_{0} ; u_{0}(0)\right)$ and

$$
M=M_{0}+1+\left|u_{0}\right|_{H}+\frac{T}{2}+\frac{1}{q}\left(\frac{T}{p C_{0}}\right)^{\frac{q}{p}}, \quad \frac{1}{p}+\frac{1}{q}=1 .
$$

We put

$$
V_{M_{0}}:=\left\{v ; v=u_{0} \text { on }\left[-\delta_{0}, 0\right], \sup _{0 \leq t \leq T}\left\{\frac{1}{2} \int_{0}^{t}\left|v^{\prime}(s)\right|_{H}^{2} d s+C_{0}|v(t)|_{X}^{p}\right\} \leq M_{0}+1\right\},
$$

which is a closed convex subset of $W^{1,2}\left(-\delta_{0}, T ; H\right) \cap L^{\infty}\left(-\delta_{0}, T ; H\right)$ and compact in $C\left(\left[-\delta_{0}, T\right] ; H\right)$, and

$$
Y_{M}:=\left\{v \in V\left(-\delta_{0}, T\right) ;|v|_{V\left(-\delta_{0}, T\right) \leq M}\right\} .
$$

By assumption (H1), there are non-negative functions $a_{M} \in L^{2}(0, T)$ and $b_{M} \in L^{1}(0, T)$ such that $\left\{\varphi^{t}(v ; \cdot)\right\}_{0 \leq t \leq T} \in G\left(a_{M}, b_{M}\right)$ for each $v \in Y_{M}$. It is easy to check by the choice of $M$ that $V_{M_{0}} \subset Y_{M}$.

Now, for each $v \in V_{M_{0}}$, we consider the evolution problem

$$
\left\{\begin{array}{l}
u^{\prime}(t)+\partial \varphi^{t}(v ; u(t)) \ni f(t) \text { a.e. } t \in[0, T] \\
u(0)=u_{0}(0)
\end{array}\right.
$$

By virtue of the result mentioned in the previous section, this problem has a unique solution $u \in W^{1,2}(0, T ; H)$ for each $v \in V_{M_{0}}$. 
Lemma 4.1. There exists a positive number $T_{1}$ such that

$$
\frac{1}{2} \int_{0}^{t}\left|u^{\prime}(\tau)\right|_{H}^{2} d \tau+C_{0}|u(t)|_{X}^{p} \leq M_{0}+1, \quad \forall t \in\left[0, T_{1}\right],
$$

for every $v \in V_{M_{0}}$ and the solution $u$ of (4.1).

Proof. Using Gronwall's lemma to the energy inequality (cf. (2.2))

$$
\varphi^{t}(v ; u(t))+\frac{1}{2} \int_{0}^{t}\left|u^{\prime}(\tau)\right|_{H}^{2} d \tau \leq \varphi^{0}\left(v ; u_{0}\right)+\int_{0}^{t} k_{M}(\tau) \cdot\left(\varphi^{\tau}(v ; u(\tau))+1\right) d \tau, \forall t \in[0, T],
$$

where $k_{M}(\tau):=6\left\{a_{M}(\tau)^{2}+b_{M}(\tau)+|f(\tau)|_{H}^{2}\right\}$, we have for all $t \in[0, T]$

$$
\begin{gathered}
\varphi^{t}(v ; u(t))+\frac{1}{2} \int_{0}^{t}\left|u^{\prime}(\tau)\right|_{H}^{2} d \tau \\
\leq \exp \left(\int_{0}^{t} k_{M}(\tau) d \tau\right) \varphi^{0}\left(u_{0} ; u_{0}(0)\right)+\exp \left(\int_{0}^{T} k_{M}(\tau) d \tau\right) \int_{0}^{t} k_{M}(\tau) d \tau \\
=\varphi^{0}\left(u_{0} ; u_{0}(0)\right)+\left(\exp \left(\int_{0}^{t} k_{M}(\tau) d \tau\right)-1\right) \varphi^{0}\left(u_{0} ; u_{0}(0)\right) \\
+\exp \left(\int_{0}^{T} k_{M}(\tau) d \tau\right) \int_{0}^{t} k_{M}(\tau) d \tau .
\end{gathered}
$$

Hence

$$
\varphi^{t}(v ; u(t))+\frac{1}{2} \int_{0}^{t}\left|u^{\prime}\right|_{H} d \tau \leq M_{0}+1, \forall t \in\left[0, T_{1}\right]
$$

choosing a small $T_{1} \in(0, T]$ with

$$
\left(\exp \left(\int_{0}^{T_{1}} k_{M}(\tau) d \tau\right)-1\right) \varphi^{0}\left(u_{0} ; u_{0}(0)\right)+\exp \left(\int_{0}^{T} k_{M}(\tau) d \tau\right) \int_{0}^{T_{1}} k_{M}(\tau) d \tau \leq 1 .
$$

Proof of Theorem 1. We define an operator $S: V_{M_{0}} \rightarrow V_{M_{0}}$ by

$$
[S v](t)=\left\{\begin{array}{lc}
u_{0}(t), & -\delta_{0} \leq t \leq 0 \\
u(t), & 0<t \leq T_{1} \\
u\left(T_{1}\right), & T_{1}<t \leq T .
\end{array}\right.
$$

where $T_{1}$ is the same number as in Lemma 4.1 and $u$ is the solution of (4.1).

Now we show that $S$ is continuous in $C\left(\left[-\delta_{0}, T\right] ; H\right)$. Take $\left\{v_{n}\right\} \subset V_{M_{0}}$ so that $v_{n} \rightarrow v$ in $C\left(\left[-\delta_{0}, T\right] ; H\right)$. By assumption $(\Phi 3)$,

$$
\varphi^{t}\left(v_{n} ; \cdot\right) \rightarrow \varphi^{t}(v ; \cdot) \text { on } H \text { in the sense of Mosco for every } t \in[0, T] .
$$

By the general convergence result mentioned in section 2, we observe that

$$
\begin{aligned}
& u_{n}:=S v_{n} \rightarrow u \text { in } C\left(\left[0, T_{1}\right] ; H\right), \\
& u_{n}^{\prime} \rightarrow u^{\prime} \text { weakly in } L^{2}\left(0, T_{1} ; H\right)
\end{aligned}
$$


and $u$ is the solution of $(4.1)$ on $\left[0, T_{1}\right]$. Therefore,

$$
[S v](t)=\left\{\begin{array}{lc}
u_{0}(t), & -\delta_{0} \leq t \leq 0, \\
u(t), & 0<t \leq T_{1}, \\
u\left(T_{1}\right), & T_{1}<t \leq T,
\end{array}\right.
$$

which shows that $S v_{n} \rightarrow S v$ in $C\left(\left[-\delta_{0}, T\right] ; H\right)$.

Hence, $S v=u$ and $S$ is continuous in $C\left(\left[-\delta_{0}, T\right] ; H\right)$. By the fixed point theorem for compact operators, $S$ has at least one fixed point $u$ in $V_{M_{0}}$. We easily check $u$ is a solution of $\operatorname{CP}\left(u_{0}, f\right)$ on the time interval $\left[0, T_{1}\right]$.

5. Proof of Theorem 2. By virtue of Theorem $1, \operatorname{CP}\left(u_{0}, f\right)$ has at least one solution $u$ on a certain time interval $\left[0, T^{\prime}\right], 0<T^{\prime} \leq T$, such that

$$
u \in W^{1,2}\left(-\delta_{0}, T^{\prime} ; H\right), \sup _{0 \leq t \leq T^{\prime}} \varphi^{t}(u ; u(t))<\infty .
$$

Put

$$
T^{*}:=\sup \left\{T^{\prime} ; \text { there exists a solution } u \text { of } \mathrm{CP}\left(u_{0}, f\right) \text { on }\left[0, T^{\prime}\right]\right\} .
$$

First we show that $\operatorname{CP}\left(u_{0}, f\right)$ has a solution on the interval $\left[0, T^{*}\right]$. By the definition of $T^{*}$, there are a sequence $\left\{T_{n}\right\}$ with $T_{n} \nearrow T^{*}$ and a sequence $\left\{u_{n}\right\}$ of solutions $u_{n}$ of $\mathrm{CP}\left(u_{0}, f\right)$ on $\left[0, T_{n}\right]$ for every $n \in \mathbb{N}$. From the energy inequality (2.1) and assumption (H2) it follows that

$$
\sup _{t \in\left[0, T_{n}\right]}\left|u_{n}(t)\right|_{H}^{2}+\int_{0}^{T_{n}} \varphi^{t}\left(u_{n} ; u_{n}(t)\right) d t \leq A_{1}, \forall n=1,2, \cdots,
$$

where $A_{1}$ is a positive constant depending only on $A_{0}$ appearing in (H2), $\left|u_{0}\right|_{H}$ and $|f|_{L^{2}(0, T ; H)}$; note that $A_{1}$ is independent of $n$. Now, define a function $\tilde{u}_{n}:\left[-\delta_{0}, T\right] \rightarrow H$ by

$$
\tilde{u}_{n}= \begin{cases}u_{0} & \text { on }\left[-\delta_{0}, 0\right), \\ u_{n} & \text { on }\left[0, T_{n}\right] \\ 0 & \text { on }\left(T_{n}, T\right]\end{cases}
$$

which clearly belongs to $L^{\infty}\left(-\delta_{0}, T ; H\right) \cap L^{p}\left(-\delta_{0}, T ; X\right)$ and satisfies that

$$
\left|\tilde{u}_{n}\right|_{L^{\infty}\left(-\delta_{0}, T ; H\right)}+\left|\tilde{u}_{n}\right|_{L^{p}\left(-\delta_{0}, T ; X\right)} \leq A_{2}:=A_{1}^{\frac{1}{2}}+\left(\frac{A_{1}}{C_{0}}\right)^{\frac{1}{p}}, \quad \forall n=1,2, \cdots .
$$

Therefore there exists a subsequence $\left\{\tilde{u}_{n_{k}}\right\}$ of $\left\{\tilde{u}_{n}\right\}$ and a function $\tilde{u}$ so that

$$
\tilde{u}_{n_{k}} \rightarrow \tilde{u} \text { weakly in } L^{p}\left(-\delta_{0}, T ; X\right) \text {, weakly } * \text { in } L^{\infty}\left(-\delta_{0}, T ; H\right) .
$$

Assumption ( $\Phi 3)$ implies that

$$
\varphi^{t}\left(\tilde{u}_{n_{k}} ; \cdot\right) \rightarrow \varphi^{t}(\tilde{u} ; \cdot) \text { on } H \text { in the sense of Mosco, } \forall t \in[0, T] .
$$

Here, apply the convergence result mentioned in section 2 to see that the solution $\tilde{w}_{k}$ of

$$
\tilde{w}_{k}^{\prime}(t)+\partial \varphi^{t}\left(\tilde{u}_{n_{k}} ; \tilde{w}_{k}(t)\right) \ni f(t), 0<t<T, \tilde{w}_{k}(0)=u_{0}(0),
$$

converges to the solution $\tilde{w}$ of

$$
\tilde{w}^{\prime}(t)+\partial \varphi^{t}(\tilde{u} ; \tilde{w}(t)) \ni f(t), 0<t<T, \tilde{w}(0)=u_{0}(0),
$$


in the sense that

$$
\begin{gathered}
\tilde{w}_{k} \rightarrow \tilde{w} \text { in } C([0, T] ; H), \tilde{w}_{k}^{\prime} \rightarrow \tilde{w}^{\prime} \text { weakly in } L^{2}(0, T ; H), \\
\sup _{k \in \mathbb{N}, t \in[0, T]} \varphi^{t}\left(\tilde{u}_{n_{k}} ; \tilde{w}_{k}(t)\right)<\infty .
\end{gathered}
$$

On the other hand, since $\tilde{u}_{n_{k}}=u_{n_{k}}=\tilde{w}_{k}$ on $\left[0, T_{n}\right]$, it follows from (5.1) and (5.2) that

$$
\begin{gathered}
u_{n_{k}}\left(T_{n_{k}}\right) \rightarrow \tilde{w}\left(T^{*}\right) \text { in } H \\
\varphi^{T^{*}}\left(\tilde{u} ; \tilde{w}\left(T^{*}\right)\right) \leq \liminf _{n \rightarrow \infty} \varphi^{T_{n_{k}}}\left(\tilde{u}_{n_{k}} ; \tilde{w}_{k}\left(T_{n_{k}}\right)\right) \\
\left(=\liminf _{n \rightarrow \infty} \varphi^{T_{n_{k}}}\left(u_{n_{k}} ; u_{n_{k}}\left(T_{n_{k}}\right)\right)\right) \\
<\infty .
\end{gathered}
$$

Hence $\tilde{u}=\tilde{w}$ a.e. on $\left(0, T^{*}\right)$, which shows that $\tilde{u}$ is a solution of $\operatorname{CP}\left(u_{0}, f\right)$ on $\left[0, T^{*}\right]$ such that $\tilde{u} \in W^{1,2}\left(-\delta_{0}, T^{*} ; H\right), \sup _{0 \leq t \leq T^{*}} \varphi^{t}(\tilde{u} ; \tilde{u}(t))<\infty$.

Next, assume that $T^{*}<T$. Then, applying Theorem 1 to the problem with initial time $T^{*}$ and initial datum $\tilde{u}_{0}=\left.u\right|_{\left[-\delta_{0}, T^{*}\right]}$, we obtain an extension of $\tilde{u}$ beyond time $T^{*}$ as a solution of $\operatorname{CP}\left(u_{0}, f\right)$. This contradicts the definition of $T^{*}$. Thus $\operatorname{CP}\left(u_{0}, f\right)$ must have a solution on the whole time interval $[0, T]$.

6. Applications. We give some applications of Theorem 2 to parabolic quasi-variational inequalities.

Let $\Omega$ be a bounded domain in $\mathbf{R}^{N}, 1 \leq N<\infty$, with smooth boundary $\Gamma:=$ $\partial \Omega$. Furthermore let $k_{c}(\cdot)$ be a Lipschitz continuous function on $\mathbf{R}$ with bounded Lipschitz continuous derivative $k_{c}^{\prime}(\cdot)$ on $\mathbf{R}$. Let $\delta_{0}>0, f \in L^{2}\left(0, T ; L^{2}(\Omega)\right)$, and $u_{0} \in$ $L^{2}\left(-\delta_{0}, 0 ; H^{1}(\Omega)\right) \cap W^{1,2}\left(-\delta_{0}, 0 ; L^{2}(\Omega)\right)$.

EXAMPLE 6.1. We consider the following evolution problem:

$$
\left\{\begin{array}{l}
u \in W^{1,2}\left(-\delta_{0}, T ; L^{2}(\Omega)\right) \cap L^{\infty}\left(-\delta_{0}, T ; H^{1}(\Omega)\right), \\
u \geq k_{c}(\Lambda u) \text { a.e. on } \Omega \times(0, T), \\
\int_{0}^{T} \int_{\Omega}\left(u_{t}-f\right)(u-w) d x d t+\int_{0}^{T} \int_{0}^{1} \nabla u(\nabla u-\nabla w) d x d t \leq 0, \\
\quad \forall w \in L^{2}\left(0, T ; H^{1}(\Omega)\right) \text { with } w \geq k_{c}(\Lambda u) \text { a.e. on } \Omega \times(0, T), \\
u=u_{0} \text { a.e. on } \Omega \times\left[-\delta_{0}, 0\right] ;
\end{array}\right.
$$

where

$$
[\Lambda v](x, t)=\int_{-\delta_{0}}^{t} \int_{\Omega} \rho(x, y, s, t) v(y, s) d y d s, \forall(x, t) \in \Omega \times[0, T],
$$

for a bounded smooth function $\rho$ on $\bar{\Omega} \times \bar{\Omega} \times\left[-\delta_{0}, T\right] \times[0, T]$.

We apply Theorem 2 to the case when $H=L^{2}(\Omega), X=H^{1}(\Omega)$ and

$$
\varphi^{s}(v ; u):=\frac{1}{2} \int_{\Omega}|\nabla u|^{2} d x+\int_{\Omega} I_{K(s ; v)}(u) d x, \forall u \in X, \forall 0 \leq s \leq t,
$$


where

$$
K(s ; v):=\left\{w \in X \mid w \geq k_{c}([\Lambda v](s)) \text { a.e. on } \Omega\right\}, \forall 0 \leq s \leq t,
$$

and $I_{K(s ; v)}(\cdot)$ is the indicator function of $K(s ; v)$, i.e. $I_{K(s ; v)}$ is a proper l.s.c. convex function on $H$ defined by

$$
I_{K(s ; v)}(z)=\left\{\begin{array}{cc}
0, & z \in K(s ; v), \\
\infty, & z \notin K(s ; v) .
\end{array}\right.
$$

It is easy to see that $\varphi^{s}(v ; \cdot)$ satisfies $(\Phi 1),(\Phi 2)$ and $(\Phi 3)$.

LEMma 6.1. For each $M>0$, there exists a non-negative function $a_{M}\left(=b_{M}\right)$ in $L^{2}(0, T)$ such that (H1) and (H2) hold.

Proof. For clearly $0 \leq s \leq t_{1} \leq t, v \in V_{M}$ and $z \in D\left(\varphi^{s}(v ; \cdot)\right)=K(s ; v)$, consider

$$
\tilde{z}:=z-k_{c}(\Lambda v(s))+k_{c}\left(\Lambda v\left(t_{1}\right)\right)
$$

Then, $\tilde{z} \in K\left(t_{1} ; v\right)=D\left(\varphi^{t_{1}}(v ; \cdot)\right)$.

Moreover, with Lipschitz constant $L\left(k_{c}\right)$ and $L(\rho)$ of $k_{c}$ and $\rho$, respectively, we have

$$
\begin{aligned}
|\tilde{z}-z|_{L^{2}(\Omega)} & =\left|k_{c}\left(\Lambda v\left(t_{1}\right)\right)-k_{c}(\Lambda v(s))\right|_{L^{2}(\Omega)} \\
& \leq \int_{s}^{t_{1}} L\left(k_{c}\right)\left|(\Lambda v)^{\prime}(\tau)\right|_{L^{2}(\Omega)} d \tau \\
& \leq \int_{s}^{t_{1}} L\left(k_{c}\right)(L(\rho)+\max |\rho|)|v|_{L^{\infty}\left(-\delta_{0}, T ; L^{2}(\Omega)\right)} d \tau \\
& \leq C_{2} M\left|t_{1}-s\right|,
\end{aligned}
$$

where $C_{2}=L\left(k_{c}\right)(L(\rho)+\max |\rho|)$. Similarly, for a constant $C_{3}>0$ we have

$$
\begin{aligned}
& |\nabla \tilde{z}|_{L^{2}(\Omega)}^{2}-|\nabla z|_{L^{2}(\Omega)}^{2} \\
& \leq\left|\nabla\left(k_{c}\left(\Lambda v\left(t_{1}\right)\right)-k_{c}(\Lambda v(s))\right)\right|_{L^{2}(\Omega)}^{2}+2|\nabla z|_{L^{2}(\Omega)}\left|\nabla\left(k_{c}\left(\Lambda v\left(T_{1}\right)\right)-k_{c}(\Lambda v(s))\right)\right|_{L^{2}(\Omega)} \\
& \leq C_{3} M\left|t_{1}-s\right| \cdot\left(\frac{1}{2}|\nabla z|_{L^{2} \Omega}^{2}+1\right) .
\end{aligned}
$$

Therefore, it is enough to choose $a_{M}(t):=C_{4} M$ for $0 \leq t \leq T, C_{4}=C_{2}+C_{3}$.

EXAMPLE 6.2. We consider the following evolution problem:

$$
\left\{\begin{array}{l}
u \in W^{1,2}\left(-\delta_{0}, T ; L^{2}(\Omega)\right) \cap L^{\infty}\left(-\delta_{0}, T ; H^{1}(\Omega)\right), \\
u \geq k_{c}(\Lambda u) \text { a.e. on } \Gamma \times(0, T), \\
\int_{0}^{T} \int_{\Omega}\left(u_{t}-f\right)(u-w) d x d t+\int_{0}^{T} \int_{0}^{1} \nabla u(\nabla u-\nabla w) d x d t \leq 0, \\
\quad \forall w \in L^{2}\left(0, T ; H^{1}(\Omega)\right) \text { with } w \geq k_{c}(\Lambda u) \text { a.e. on } \Gamma \times(0, T), \\
u=u_{0} \text { a.e. on } \Gamma \times\left[-\delta_{0}, 0\right],
\end{array}\right.
$$


where

$$
\begin{array}{r}
{[\Lambda v](x, t):=\int_{-\delta_{0}}^{t} \int_{\Gamma} \rho(x, y, s, t) v(y, s) d \Gamma d s, \forall(x, t) \in \Gamma \times[0, T]} \\
\forall v \in L^{2}\left(-\delta_{0}, t ; L^{2}(\Omega)\right), 0<t \leq T .
\end{array}
$$

We use the following notation:

$$
\begin{aligned}
& K(s ; v):=\left\{w \in H^{1}(\Omega) \mid w \geq k_{c}([\Lambda v](s)) \text { a.e. on } \Gamma\right\}, \\
& \varphi^{s}(v ; u):=\frac{1}{2} \int_{\Omega}|\nabla u|^{2} d x+\int_{\Omega} I_{K(s ; v)}(u) d x, \forall u \in H^{1}(\Omega),
\end{aligned}
$$

for $0 \leq s \leq t \leq T, v \in L^{2}\left(-\delta_{0}, t ; L^{2}(\Omega)\right)$.

In this case, (H1) and (H2) are easily verified too. Therefore, problem (6.1) has a solution $u$.

EXAMPLE 6.3. We consider the following evolution problem:

$$
\left\{\begin{array}{c}
u \in W^{1,2}\left(-\delta_{0}, T ; L^{2}(0,1)\right) \cap L^{\infty}\left(-\delta_{0}, T ; H^{1}(0,1)\right), \\
\left|u_{x}\right| \leq k_{c}(\Lambda u) \text { a.e. on }(0,1) \times(0, T), u(0, t)=0 \text { for } t \in[0, T], \\
\int_{0}^{T} \int_{0}^{1}\left(u_{t}-f\right)(u-w) d x d t+\int_{0}^{T} \int_{0}^{1} u_{x}\left(u_{x}-w_{x}\right) d x d t \leq 0, \\
\forall w \in L^{2}\left(0, T ; H^{1}(0,1)\right) \text { with }\left|w_{x}\right| \leq k_{c}(\Lambda u) \text { a.e. on }(0,1) \times(0, T), \\
w(0, t)=0 \text { for a.e. } t \in(0, T), \\
u=u_{0} \text { on }(0,1) \times\left[-\delta_{0}, 0\right]
\end{array}\right.
$$

where

$$
[\Lambda v](x, t):=\int_{-\delta_{0}}^{t} \int_{0}^{1} \rho(x, y, s, t) v(y, s) d y d s, \forall(x, t) \in(0,1) \times[0, T]
$$

for a bounded smooth function $\rho$ on $[0,1] \times[0,1] \times\left[-\delta_{0}, T\right] \times[0, T]$.

We apply Theorem 2 in the case when $H=L^{2}(0,1), X=\left\{z \in H^{1}(0,1) ; z(0)=0\right\}$ and put

$$
\varphi^{s}(v ; u):=\frac{1}{2} \int_{0}^{1}\left|u_{x}\right|^{2} d x+\int_{0}^{1} I_{K(s ; v)}(u) d x, \forall u \in X, \forall 0 \leq s \leq t
$$

where

$$
K(s ; v):=\left\{w \in X|| w_{x} \mid \leq k_{c}([\Lambda v](s)) \text { a.e. on }(0,1)\right\}, \forall 0 \leq s \leq t,
$$

and $I_{K(s ; v)}(\cdot)$ is the indicator function of $K(s ; v)$.

We easily see that the convex function $\varphi^{s}(v ; \cdot)$ satisfies $(\Phi 1),(\Phi 2),(\Phi 3)$.

Lemma 6.2. For each $M>0$, there exists a non-negative function $a_{M}\left(=b_{M}\right)$ in $L^{2}(0, T)$, such that (H1) and (H2) hold. 
Proof. For $0 \leq s \leq t_{1} \leq t, v \in V\left(-\delta_{0}, t\right)$ with $|v|_{V\left(-\delta_{0}, t\right)} \leq M, z \in D\left(\varphi^{s}(v ; \cdot)\right)=K(s ; v)$, put

$$
\tilde{z}(x):=\frac{k_{c}\left([\Lambda v]\left(x, t_{1}\right)\right)}{k_{c}([\Lambda v](x, s))} z(x)-\int_{0}^{x}\left(\frac{k_{c}\left([\Lambda v]\left(y, t_{1}\right)\right)}{k_{c}([\Lambda v](y, s))}\right)_{y} z(y) d y .
$$

Then

$$
\left|\tilde{z}_{x}\right|=\left|\frac{k_{c}\left([\Lambda v]\left(x, t_{1}\right)\right)}{k_{c}([\Lambda v](x, s))}\right|\left|z_{x}(x)\right| \leq k_{c}\left([\Lambda v]\left(x, t_{1}\right)\right)
$$

hence, $\tilde{z} \in K\left(t_{1} ; v\right)=D\left(\varphi^{t_{1}}(v ; \cdot)\right)$.

Furthermore, for some positive constants $C_{5} \sim C_{8}$, we have

$$
|\tilde{z}-z|_{L^{2}(0,1)} \leq\left\{C_{5}\left(M\left|t_{1}-s\right|\right)^{2} \cdot|z|_{L^{2}(0,1)}^{2}\right\}^{\frac{1}{2}} \leq C_{6} M\left|t_{1}-s\right||z|_{L^{2}(0,1)} .
$$

and

$$
\begin{aligned}
|\nabla \tilde{z}|_{L^{2}(0,1)}^{2}-|\nabla z|_{L^{2}(0,1)}^{2} & =\int_{0}^{1} \frac{\left|k_{c}\left(\Lambda v\left(x, t_{1}\right)\right)\right|^{2}-\left|k_{c}(\Lambda v(x, s))\right|^{2}}{\left|k_{c}(\Lambda v(x, s))\right|^{2}}\left|z_{x}(x)\right|^{2} d x \\
& \leq C_{7} \int_{0}^{1}\left|\Lambda v\left(x, t_{1}\right)-\Lambda v(x, s)\right|\left|z_{x}(x)\right|^{2} d x \\
& \leq C_{8} M\left|t_{1}-s\right|\left|z_{x}\right|_{L^{2}(0,1)}^{2}
\end{aligned}
$$

Hence, if $a_{M}(t):=C_{9} M$ for $0 \leq t \leq T, C_{9}=C_{6}+C_{8}$, then (H1) is satisfied.

In connection with semiconductor problems, a parabolic quasi-variational inequality was treated in [8], in which the so-called penalty method is employed in order to approximate the original problem.

\section{References}

[1] R. Kano, N. Kenmochi and Y. Murase, Existence theorems for elliptic quasi-variational inequalities in Banach spaces, to appear.

[2] R. Kano, N. Kenmochi and Y. Murase, Nonlinear evolution equations generated by subdifferentials with nonlocal constraints, this volume, 175-194.

[3] N. Kenmochi, Some nonlinear parabolic variational inequalities, Israel J. Math. 22 (1975), 304-331.

[4] N. Kenmochi, Solvability of nonlinear evolution equations with time-dependent constraints and applications, Bull. Fac. Education Chiba Univ. 30 (1981), 1-87.

[5] N. Kenmochi, Monotonicity and Compactness Methods for Nonlinear Variational Inequalities, Chapter 4 (pp. 203-298) in Handbook of Differential Equations, Stationary Partial Differential Equations, Vol. 4 (edited by M. Chipot), Elsevier, Amsterdam, 2007.

[6] F. Mignot and J.-P. Puel, Inéquations d'évolution paraboliques avec convexes dépendant du temps. Applications aux inéquations quasi variationnelles d'évolution, Arch. Rat. Mech. Anal. 64 (1977), 59-91.

[7] U. Mosco, Convergence of convex sets and of solutions of variational inequalities, Advances in Math. 3 (1969), 510-585.

[8] J. F. Rodrigues and L. Santos, A parabolic quasi-variational inequality arising in a superconductivity model, Ann. Scuola Norm. Sup. Pisa 29 (2000), 153-169. 
[9] U. Stefanelli, Nonlocal quasivariational evolution problems, J. Differential Equations 229 (2006), 204-228.

[10] Y. Yamada, On nonlinear evolution equations generated by the subdifferentials, J. Fac. Sci., Univ. Tokyo Sect. IA 23 (1976), 491-515. 